\title{
Complete Genome Sequence of Bacillus cereus Bacteriophage PBC1
}

\author{
Minsuk Kong, Minsik Kim, and Sangryeol Ryu \\ Department of Food and Animal Biotechnology, Department of Agricultural Biotechnology and Center for Agricultural Biomaterials, Seoul National University, Seoul, \\ South Korea
}

\begin{abstract}
Bacillus cereus is a ubiquitous, spore-forming bacterium associated with food poisoning cases. To develop an efficient biocontrol agent against $B$. cereus, we isolated lytic phage $\mathrm{PBC1}$ and sequenced its genome. $\mathrm{PBC1}$ showed a very low degree of homology to previously reported phages, implying that it is novel. Here we report the complete genome sequence of $\mathrm{PBC} 1$ and describe major findings from our analysis.
\end{abstract}

$B_{t a t a n}^{a}$ acillus cereus is an opportunistic human pathogen responsible for 2 to $5 \%$ of the food-borne illnesses reported worldwide (2, $8)$. Although outbreaks caused by B. cereus are relatively infrequent, concerns about $B$. cereus have not diminished because it is commonly isolated from foods and food additives due to its abilities to form endospores and to grow at a broad range of temperatures $\left(5\right.$ to $\left.55^{\circ} \mathrm{C}\right)(5,9,10,15)$. With the rapid emergence of antibiotic-resistant bacteria, the use of bacteriophages has regained attention as an efficient alternative method for their control $(7,14)$. Although many phages infecting various strains of $B$. cereus have been isolated in our lab $(11,16)$, only one phage, PBC1, can infect B. cereus strain ATCC 21768. PBC1 belongs to the Siphoviridae family and forms clear plaques (data not shown).

Phenol-chloroform extraction was used to isolate the phage's genomic DNA, and it was sequenced using the Genome Sequencer FLX Titanium by Macrogen, Seoul, South Korea. The assembly of quality filtered reads was performed using GS De Novo Assembler (v. 2.6), and the open reading frames (ORFs) were predicted using the Glimmer 3.02 (4), GeneMark.hmm (3), and FgenesB (Softberry, Inc., Mount Kisco, NY) software. The ORFs were limited to those encoding proteins of more than 50 amino acids. Conserveddomain analysis of the predicted ORFs was carried out using BLASTP (1), InterProScan (17), and the NCBI Conserved Domain Database (13). Searches for tRNAs were conducted using the tRNAscan-SE program (12).

Genomic analysis revealed that PBC1 contains 41,164 bp of linear double-stranded DNA with a G+C content of 41.7 mol\%. $\mathrm{PBC} 1$ has terminally redundant and partially permuted genomes, suggesting that PBC1 uses a headful packaging mechanism. We identified 50 predicted ORFs, all of which were transcribed in the same direction, and found no tRNAs. Of the 50 predicted ORFs, 28 were identified as encoding hypothetical proteins. Homology searches identified packaging and structural proteins (a terminase, a portal protein, major/minor capsid proteins, and a tail length measure protein), host lysis proteins (a holin and an endolysin), and DNA replication and modification proteins (a thymidylate synthase, a nucleoside triphosphatase, a DNA polymerase, a resolvase, a glutaredoxin-like protein, a nuclease, and a helicase). We could not find any lysogeny-related proteins, such as an integrase or repressors, supporting the notion that $\mathrm{PBC} 1$ is a virulent phage. Interestingly, $\mathrm{PBC} 1$ has a putative YD repeat protein at its tail that is known to be involved in extracellular carbohydrate binding (6). We speculate that the host, B. cereus ATCC 21768 , may have a unique carbohydrate structure on the cell surface and that $\mathrm{PBC} 1$ specifically recognizes and binds the host receptor by using the YD repeat protein. The analysis of the complete $\mathrm{PBC} 1$ genome not only facilitates its development as a biocontrol agent against $B$. cereus but also improves our understanding of the bacteriophage host range.

Nucleotide sequence accession number. The complete genome sequence of $B$. cereus phage PBC1 is available in GenBank under accession number JQ619704.

\section{ACKNOWLEDGMENT}

This work was supported by the Agriculture Research Center program of the Ministry for Food, Agriculture, Forestry and Fisheries, Republic of Korea.

\section{REFERENCES}

1. Altschul SF, Gish W, Miller W, Myers EW, Lipman DJ. 1990. Basic local alignment search tool. J. Mol. Biol. 215:403-410.

2. Anonymous. 2011. Surveillance for foodborne disease outbreaksUnited States, 2008. MMWR Morb. Mortal. Wkly. Rep. 60:1197-1202.

3. Besemer J, Lomsadze A, Borodovsky M. 2001. GeneMarkS: a selftraining method for prediction of gene starts in microbial genomes. Implications for finding sequence motifs in regulatory regions. Nucleic Acids Res. 29:2607-2618.

4. Delcher AL, Bratke KA, Powers EC, Salzberg SL. 2007. Identifying bacterial genes and endosymbiont DNA with Glimmer. Bioinformatics 23:673-679.

5. de Sarrau B, et al. 2012. Influence of anaerobiosis and low temperature on Bacillus cereus growth, metabolism, and membrane properties. Appl. Environ. Microbiol. 78:1715-1723.

6. Feulner G, et al. 1990. Structure of the rhsA locus from Escherichia coli $\mathrm{K}-12$ and comparison of rhs A with other members of the rhs multigene family. J. Bacteriol. 172:446-456.

7. Hagens S, Loessner MJ. 2007. Application of bacteriophages for detection and control of foodborne pathogens. Appl. Microbiol. Biotechnol. 76: 513-519.

8. Ivanova N, et al. 2003. Genome sequence of Bacillus cereus and comparative analysis with Bacillus anthracis. Nature 423:87-91.

9. Kailas L, et al. 2011. Surface architecture of endospores of the Bacillus cereus/anthracis/thuringiensis family at the subnanometer scale. Proc. Natl. Acad. Sci. U. S. A. 108:16014-16019.

10. Kim SK, et al. 2009. Prevalence and toxigenic profiles of Bacillus cereus isolated from dried red peppers, rice, and Sunsik in Korea. J. Food Prot. 72:578-582.

11. Lee JH, Shin H, Son B, Ryu S. 2012. Complete genome sequence of Bacillus cereus bacteriophage BCP78. J. Virol. 86:637-638.

Received 21 March 2012 Accepted 21 March 2012 Address correspondence to Sangryeol Ryu, sangryu@snu.ac.kr. Copyright $\odot$ 2012, American Society for Microbiology. All Rights Reserved. doi:10.1128/JVI.00706-12 
12. Lowe TM, Eddy SR. 1997. tRNAscan-SE: a program for improved detection of transfer RNA genes in genomic sequence. Nucleic Acids Res. 25: 955-964.

13. Marchler-Bauer A, et al. 2007. CDD: a conserved domain database for interactive domain family analysis. Nucleic Acids Res. 35:D237-D240.

14. Matsuzaki S, et al. 2005. Bacteriophage therapy: a revitalized therapy against bacterial infectious diseases. J. Infect. Chemother. 11:211-219.

15. Okanlawon BM, Ogunbanwo ST, Okunlola AO. 2010. Growth of Bacil- lus cereus isolated from some traditional condiments under different regimens. Afr. J. Biotechnol. 9:2129-2135.

16. Shin H, Bandara N, Shin E, Ryu S, Kim KP. 2011. Prevalence of Bacillus cereus bacteriophages in fermented foods and characterization of phage JBP901. Res. Microbiol. 162:791-797.

17. Zdobnov EM, Apweiler R. 2001. InterProScan—an integration platform for the signature-recognition methods in InterPro. Bioinformatics 17: 847-848. 